\title{
Direction-Selective Ganglion Cells Show Symmetric Participation in Retinal Waves During Development
}

\author{
Justin Elstrott and Marla B. Feller \\ Department of Molecular and Cell Biology and the Helen Wills Neurosciences Institute, University of California, Berkeley, Berkeley, California 94720
}

\begin{abstract}
Direction-selective ganglion cells (DSGCs) fire robustly for stimuli moving along one direction of motion and are strongly inhibited by stimuli moving in the opposite, or null, direction. In contrast to direction-selective neurons in primary visual cortex, a role for neural activity in the development of direction-selective retinal circuits has not been established. Direction-selective responses are detected at eye opening, before which spontaneous correlated activity known as retinal waves provide directional input to ganglion cells. Indeed, we observed a significant bias in wave propagation along the nasal over temporal direction. Using simultaneous calcium imaging and cell-attached recordings from three genetically labeled DSGC types in mice, we observed that all three DSGC types fire action potentials during retinal waves. However, we found that the direction of wave propagation did not influence DSGC spiking. These results indicate that the mechanisms guiding the formation of the asymmetric inhibition underlying direction selectivity in the retina are not dependent upon the directional properties of retinal waves.
\end{abstract}

\section{Introduction}

Although the mechanisms generating asymmetric responses in direction-selective ganglion cells (DSGCs) have received much attention, the processes underlying their development are not known. In the retina, stimuli moving in the preferred direction evoke robust action-potential firing from DSGCs, whereas responses to stimuli moving in the null direction are suppressed by inhibition (Taylor and Vaney, 2003). Recent work has shown that DSGCs develop normally in dark-reared animals, suggesting a limited role for visual experience (Chan and Chiao, 2008; Elstrott et al., 2008; Chen et al., 2009; Yonehara et al., 2009). In mice, these directional responses are detected at the emergence of photoreceptor-driven light responses on postnatal day (P) 11 (Chen et al., 2009), roughly $2 \mathrm{~d}$ before eye opening. Thus, the inhibition underlying direction selectivity forms early in development, before the earliest light responses.

Though vision is not necessary for the development of direction selectivity, spontaneous activity may play a role. Before vision, retinal waves provide the structured activity critical for refining the initially coarse retinal ganglion cell (RGC) projections to thalamus and superior colliculus (for review, see Torborg and Feller, 2005; Huberman et al., 2008). Recently, a role for retinal waves in the development of retinal circuits has been reported (Xu et al., 2010).

How might retinal waves help establish direction selectivity in the retina? Propagating, nonsynchronous activity is required for

Received May 5, 2010; revised June 28, 2010; accepted July 1, 2010.

This work was supported by National Institutes of Health Grants R01EY013528 and ARRAEYE019498, and National Science Foundation Grant IOS-0818983. The JamB mice were a generous gift from Dr. Joshua Sanes. The Spig1 mice were a generous gift from Dr. Masaharu Noda. We thank Dr. A. Blankenship for comments on the manuscript. We thank K. Ford for assistance with image analysis.

Correspondence should be addressed to Marla B. Feller, Department of Molecular and Cell Biology and Helen Wills Neuroscience Institute, University of California, Berkeley, 142 Life Sciences Addition-3200, Berkeley, CA 94720. E-mail:mfeller@berkeley.edu.

DOI:10.1523/JNEUROSCI.2302-10.2010

Copyright $\odot 2010$ the authors $\quad 0270-6474 / 10 / 3011197-05 \$ 15.00 / 0$ the normal development of directional circuits in ferret visual cortex (Li et al., 2008; for review, see Elstrott and Feller, 2009), and is capable of inducing direction selectivity in Xenopus optic tectum (Engert et al., 2002). Retinal waves activate starburst amacrine cells, an inhibitory interneuron critical for the computation of direction selectivity, during the first and second postnatal weeks (Zheng et al., 2004; Wang et al., 2007). In addition, retinal waves provide two sources of asymmetric activity. First, retinal waves are inherently directional with well defined wave fronts up to approximately P11. Second, retinal waves have a significant bias in wave propagation direction during the first postnatal week, which was recently described using a large-scale multielectrode array (Stafford et al., 2009). However, direction selectivity develops normally in knock-out mice lacking the $\beta 2$ subunit of the neuronal nicotinic receptors (Elstrott et al., 2008), which have altered spontaneous firing patterns that lack this directional bias (Stafford et al., 2009). The propagation bias of retinal waves during the second postnatal week, during which the first light responses are established, and their role in the establishment of directional circuits are unknown.

Here we combine calcium imaging and targeted cell-attached recordings from three genetically labeled DSGCs to characterize the directional bias in waves during the second postnatal week and to determine whether the propagation bias in retinal waves influences the firing patterns of direction-selective ganglion cells before eye opening.

\section{Materials and Methods}

Mice. All animal procedures were approved by the University of California, Berkeley Animal Care and Use Committees and conformed to the National Institutes of Health Guide for the Care and Use of Laboratory Animals, the Public Health Service Policy, and the Society for Neuroscience Policy on the Use of Animals in Neuroscience Research. We used male and female mice from three transgenic lines. All transgenic mouse lines used for this study were bred into a C57BL/6 background. The dopamine receptor 4 promoter (DRD4) mice, which label On-Off 
DSGCs (Huberman et al., 2009), the SPARCrelated protein containing Ig domain 1 promoter (Spig1) mice, which label On-DSGCs (Yonehara et al., 2008, 2009), and the junctional adhesion molecule B promoter (JamB) mice, in which Cre is expressed under the control of a tamoxifen-activated promoter and label Off-DSGCs (Kim et al., 2008), were as previously described. Jam-B mice were paired with Thyl-STOP-YFP reporter mice (Kim et al., 2008). The offspring of this pairing were given intraperitoneal injections of tamoxifen either at P1 (100 $\mu \mathrm{g}$ of tamoxifen dissolved in corn oil at $10 \mathrm{mg} / 1 \mathrm{ml}$ ) to drive expression of YFP for P4 experiments, or at P5 and P7 (1 mg dissolved in corn oil at $20 \mathrm{mg} / 1 \mathrm{ml}$ ) to drive expression of YFP for P10 experiments.

Calcium imaging and physiology. Retinas were isolated and images of cell fills were acquired on a two-photon confocal microscope as described previously (Wei et al., 2010). The YFP expression in the Off-DSGC dendrites was sufficiently bright to allow for direct imaging with the laser tuned to $920 \mathrm{~nm}$ without filling the cells.

Retinas were bulk loaded with the calcium indicator Oregon Green 488 BAPTA-1 AM (OGB-1 AM; Invitrogen) using the multicell bolus loading technique (Stosiek et al., 2003) adapted for retinal preparations (Blankenship et al., 2009). Epifluorescent calcium imaging was performed on a BX61WI microscope using a $20 \times$ water-immersion objective (UMPlanFl; Olympus), with illumination provided by a Sutter Lambda LS and controlled by a shutter (Uniblitz). Images were acquired at $2 \mathrm{~Hz}$ in MetaMorph (Universal Imaging).

GFP+ cells (DRD4 and SPIG1 mice) in OGB-1 AM-loaded retinas were targeted for cellattached recordings using a CFP filter set (49001; Chroma), which preferentially excites GFP over OGB-1 AM. In the JamB mice, YFP expression was sufficiently strong to visualize the cells in loaded retinas using the YFP filter set (49003; Chroma). Once a fluorescent cell had been identified, the inner limiting membrane over the cell was removed with a glass recording pipette and the RGC was targeted under the control of a micromanipulator (MP-225; Sutter Instruments). Recording pipettes (Garner Glass or Sutter Instruments) were pulled with a tip resistance of 3-5 M $\Omega$ (PP-830; Narishige).

To record $\mathrm{Cl}^{-}$-mediated currents during retinal waves, we conducted whole-cell voltageclamp recordings as described previously (Blankenship et al., 2009). GABAergic currents were blocked using 2-5 $\mu \mathrm{M}$ gabazine (Tocris Bioscience). For cell-attached recordings, a 150 $\mathrm{mm} \mathrm{NaCl}$ internal was used. Cell-attached data were sampled at $10 \mathrm{kHz}$ and bandpass filtered between 80 and $2000 \mathrm{~Hz}$. Spike times were extracted using custom code (Matlab; Mathworks).

Calcium imaging analysis. All imaging analysis was performed using custom code (Matlab). To detect waves, the fractional change in fluorescence intensity $\Delta F / F$ for each frame was calculated as follows:

$$
\frac{\Delta F}{F}=\frac{F_{\text {avg }}}{F_{\text {filtavg }}}-1,
$$

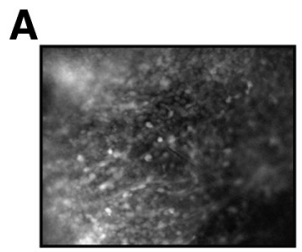

$1.5 \mathrm{~s}$

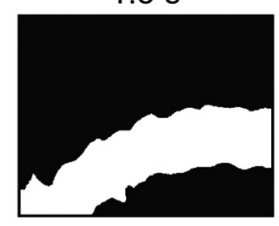

B

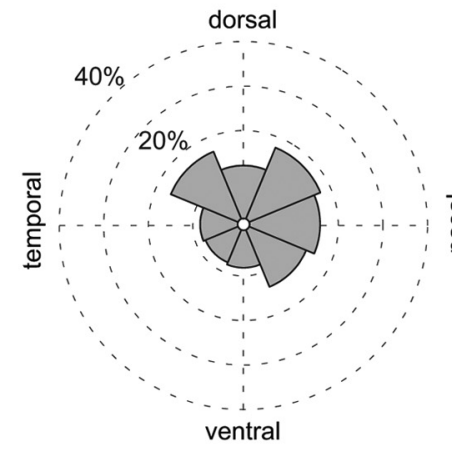

C

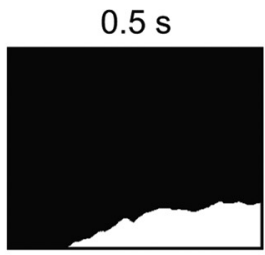

$2.0 \mathrm{~s}$
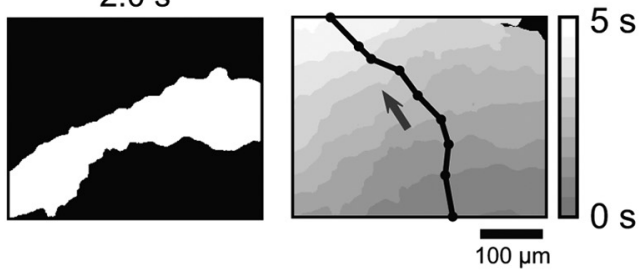

$1.0 \mathrm{~s}$

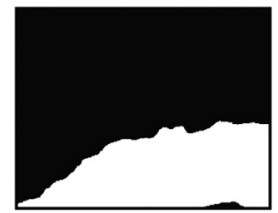

P9-10 
A
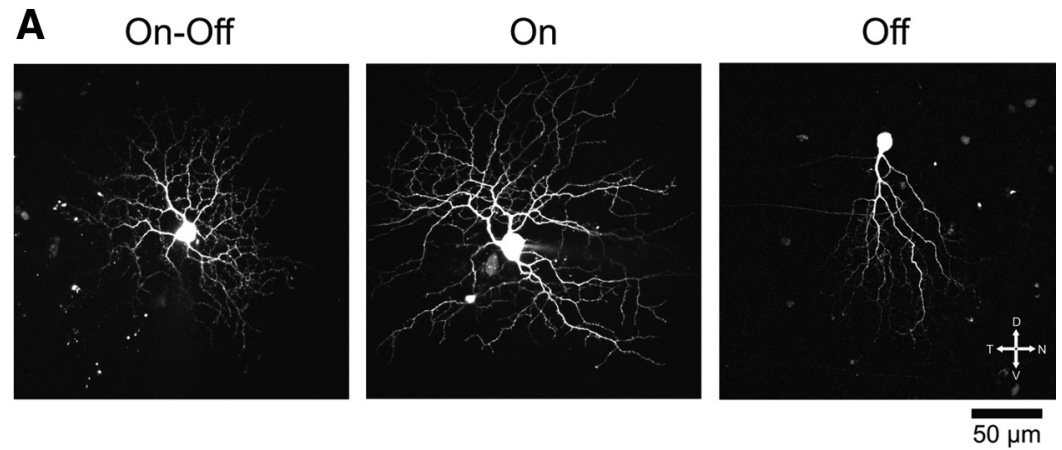

B
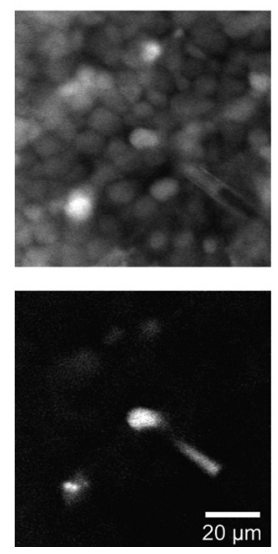

C

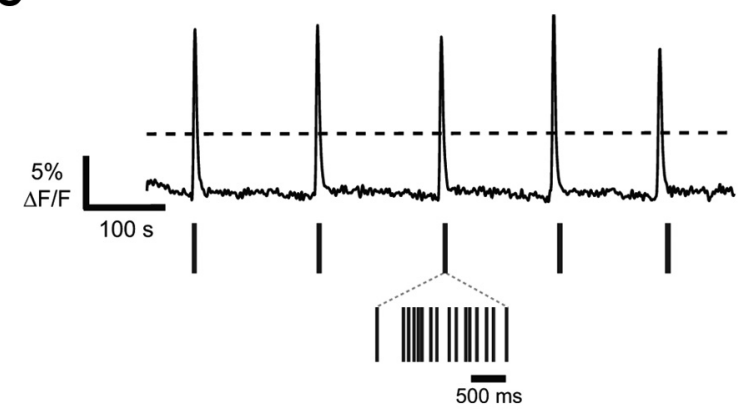

Figure 2. Characterizing DSGC participation in retinal waves using calcium imaging and cell-attached recordings. $\boldsymbol{A}$, Live two-photon images of a P10 0n-0ff-DSGC (left), a P9 0n-DSGC (middle), and a P11 Off-DSGC (right), all before eye opening. The On-Off- and 0n-DSGCs were filled with Alexa 594 before imaging. $B$, Top, Labeled somas in a P5 Spig1 retina (which express GFP in On-DSGCS) bolus loaded with the calcium indicator OGB-1 AM, imaged with a GFP cube. Bottom, The same region imaged with a CFP cube, which preferentially activates GFP over OGB-1 AM. A GFP + cell is shown in the cell-attached recording configuration. The membrane of the GFP + cell is visible within the electrode. C, Top, The $\Delta F / F$ trace reveals periodic retinal waves passing over a $200 \times 200 \mu \mathrm{m}$ region centered on the GFP + cell shown in $A$. Dashed line indicates the threshold used for isolating waves. Middle, Spike bursts elicited from the GFP + cell during waves. Inset shows the spikes for a single wave.

$\mu \mathrm{m}$ window centered on the DSGC were excluded from subsequent analysis. Next, the image frames for each retinal wave were convolved with a $20 \times 20 \mu \mathrm{m}$ spatial averaging filter. Finally, binarized image frames were obtained by applying a 2 SD intensity threshold.

Wave speed and direction were measured by connecting the nearest points of the binarized wave front across subsequent frames (Blankenship et al., 2009), which generates a direction vector on each image frame. The sum of these vectors indicates the wave direction. A wave's speed was determined by the median length of its direction vectors. The spike count for each wave was obtained by summing all of the spikes that occurred in an $8 \mathrm{~s}$ window centered on the time of threshold crossing in the $\Delta F / F$ trace.

Statistics. All statistics were performed using commercially available algorithms (Matlab). Distributions were first tested for normality using the Shapiro-Wilk test. Multiple comparisons between normal distributions were performed using one-way ANOVAs with Tukey-Kramer post hoc tests. For non-normal distributions, the Kruskal-Wallis test was used with Dunn post hoc tests for multiple comparisons, and the Wilcoxon rank sum test was used for pairwise comparisons. The $95 \%$ confidence intervals for medians were generated by resampling the original distributions and applying the bias-corrected percentile method (Efron and Tibshirani, 1986). Results with $p<0.05$ were considered significant.

\section{Results}

To test the hypothesis that retinal waves provide an instructional signal for the establishment of directional circuits, we first set out to describe the propagation bias of retinal waves. Calcium imaging was used to monitor retinal waves. Retinas were bolus loaded with the calcium-indicator dye OGB-1AM (Stosiek et al., 2003;
Blankenship et al., 2009). Binarized images of retinal waves were used to determine the propagation direction and speed for each wave (Fig. 1A) (Blankenship et al., 2009).

During the first 10 postnatal days, retinal waves exhibit well defined wavefronts (Fig. $1 A$ ). We found, consistent with previous recordings using multielectrode arrays during the first postnatal week in mouse retina (Stafford et al., 2009), a bias observed with calcium imaging toward waves propagating in the nasal direction at P3-P4 (Fig. 1 $B$, left). This bias was more pronounced at P9-P10 (Fig. $1 B$, right). The direction of the bias we observed differs slightly from that previously reported (Stafford et al., 2009). This small discrepancy may be due to the different methods used to define the direction of the wavefront using a multielectrode array versus calcium imaging. Hence, retinal waves provide a consistent directional bias in both the first and second postnatal weeks.

Interestingly, waves propagate at $\sim 100$ micrometers/s at P3-P5 and P9-P10 (Fig. $1 C)$. This corresponds to 3.3 degrees/s for visual stimuli, which is the lower bound for eliciting directional responses in adult mice (Weng et al., 2005). Hence, retinal waves may potentially act as a proxy for moving visual stimuli that activate directional circuits.

To measure the action-potential firing of DSGCs during retinal waves, we conducted simultaneous calcium imaging and physiological recordings from transgenic mice expressing XFP in particular subtypes of DSGCs (Fig. $2 A)$. Recordings were conducted from three types of transgenic mice. Expression of GFP under the DRD4 promoter uniquely labels a subtype of On-Off-DSGCs that prefer motion in the nasal direction (Fig. 2A, left) (Huberman et al., 2009). GFP expression under the SPIG1 promoter labels the On-DSGCs that prefer motion in the ventral direction (Fig. 2A, middle) (Yonehara et al., 2008, 2009). Expressing YFP under the JamB promoter (Kim et al., 2008) labels a recently discovered Off-direction-selective cell also preferring motion in the ventral direction (Fig. $2 \mathrm{~A}$, right). All three labeled DSGCs have well defined null directions in adults, allowing us to examine the influence of wave direction on DSGC responses (Figs. $2 B, C$ ).

In On-Off- and On-DSGCs, direction selectivity arises, at least in part, from asymmetric GABAergic inputs onto the cells (Fried et al., 2002; Taylor and Vaney, 2002; Sun et al., 2006). We found that GABAergic inputs were present in On-Off- and On-DSGCs during retinal waves as early as $\mathrm{P} 4$ (supplemental Fig. 1, available at www.jneurosci.org as supplemental material). We therefore examined whether these nascent GABAergic inputs translated to differences in spiking output for waves propagating in the preferred and null directions of each DSGC type. We first tested whether the direction of wave propagation influenced DSGC spiking during the first postnatal week (P3-P5), when retinal waves are mediated by a circuit composed of RGCs and starburst 
amacrine cells, the cell class critical for computing directional responses in adult On-Off- and On-DSGCs (Yoshida et al., 2001; for review, see Fried and Masland, 2007). At this age, nasal-preferring OnOff-DSGCs showed low spike counts during waves, with some cells not spiking at all ( 3 of 8 cells). Nasal-preferring On-OffDSGCs exhibited the same spiking levels regardless of whether waves moved in the preferred or null direction (On-Off, $p=$ 0.10 , one-way ANOVA) (Fig. $3 A$ ). Similarly, ventral-preferring On-DSGCs showed no difference in spiking as a function of wave direction $(p=0.69$, one-way ANOVA), even though their overall spiking per wave was higher than that of the OnOff-DSGCs ( $p<0.001$, rank sum). We did not perform these early-age experiments in Off-DSGCs. Thus, either retinal waves at this age do not selectively activate null-side inhibitory circuits or the asymmetric inhibitory inputs underlying direction selectivity are not yet mature.

We next tested whether the spiking of DSGCs in older animals (P9-P10) was influenced by the direction of wave propagation. At this age, activation of $\mathrm{GABA}_{\mathrm{A}}$ receptors hyperpolarizes RGCs (Zhang et al., 2006) and light-evoked directionselective responses have been detected 1-2 $\mathrm{d}$ later in both On-Off- and On-DSGCs (Chen et al., 2009; Yonehara et al., 2009).

On-Off-DSGCs showed significantly higher spike counts per wave at P9-P10 compared with P3-P5 (P9-P10: median, 23 spikes, 95\% CI, 21-24; vs P3-P5: median, 2 spikes; 95\% CI, 1-3; $p<0.001$, rank sum) (Fig. 3B), whereas the On-DSGCs had lower overall spike counts compared with younger ages (P9-P10: median, 9 spikes; 95\% CI, 6-11; vs P3-P5: median, 13 spikes; 95\% CI, 11-15; $p<0.05$, rank sum). However, there was no asymmetry in the firing as a function of wave direction at P9-P10 in either On-Off-DSGCs ( $p=$ 0.36 , one-way ANOVA) or On-DSGCs ( $p=0.46$, Kruskal-Wallis). The Off-DSGCs, in contrast to other Off cells (Kerschensteiner and Wong, 2008), showed the lowest overall median spike rate at P9P10 (median, 2.5 spikes; 95\% CI, 2-3; $p<0.05$, Kruskal-Wallis); yet, similar to the On-Off- and On-DSGCs, no asymmetry was detected in spike counts across wave directions ( $p=0.14$, one-way ANOVA).

\section{Discussion}

We found that that DSGCs fire robustly during retinal waves, independent of the direction of wave propagation. These results, combined with previous findings that direction selectivity is detected at eye opening independent of visual experience (Chan and Chiao, 2008; Elstrott et al., 2008; Chen et al., 2009; Yonehara et al., 2009), suggest that directional activity, either spontaneous or visually evoked, is not likely to provide an instructive cue for the formation of null-side inhibition in directional circuits.

The findings reported here are also consistent with a previous report combining multielectrode array recording and calcium imaging (M. L. Morgan and R. O. Wong, personal communications) and our finding that mice lacking the $\beta 2$ subunit of the neuronal nicotinic acetylcholine receptor $(\beta 2$-nAChR-KO) have normal directional selectivity at eye opening (Elstrott et al.,
2008). $\beta 2$-nAChR-KO mice, which do not exhibit normal retinal waves, have disrupted retinofugal projections and altered visual responses (for review, see Torborg and Feller, 2005; Wang et al., 2009). However, the spontaneous in vivo firing patterns of $\beta 2$ nAChR-KO retinas are not known - under some recording conditions, $\beta 2$-nAChR-KO retinas exhibit uncorrelated action potential firing (McLaughlin et al., 2003), whereas in others they exhibit propagating activity (Sun et al., 2008; Stafford et al., 2009). We did not record from DSGCs in $\beta 2$-nAChR-KO mice, but the compensatory waves observed in these mice do not have the same bias in wave propagation as waves in wild-type mice (Stafford et al., 2009) and therefore they are not likely to be instructive for the development of DSGCs.

The observation that DSGCs do not respond differentially to the direction of wave propagation has three possible interpretations. First, directional circuits are not yet mature. Although directional responses have been recorded as early as P11 in mice (Chen et al., 2009), the extent of these early directional responses and the mechanisms underlying them are not yet known. Second, the strength of the input during waves overwhelms the directionselective-specific inhibitory circuits. Third, waves do not activate the inhibitory circuits that mediate direction selectivity because they propagate at speeds that are not optimal for activating the directional circuitry. Adult DSGCs show characteristic speed tuning, with stimuli that are too fast or slow resulting in diminished direction selectivity (Oyster et al., 1972). In mice, both On-Off-DSGC and Off-DSGC directional responses have been reported for stimuli moving at a few degrees per second with increasing direction selectivity as the stimulus speed increases (Fig. 1C) (Weng et al., 2005; Kim et al., 2008). On-DSGCs have been 
successfully stimulated at $\sim 10 \mathrm{deg} / \mathrm{s}$ (Sun et al., 2006; Yonehara et al., 2009), though the entire tuning range has not been described. Retinal waves at P9-P10 ranged in speed from 0.6 to $14.8 \mathrm{deg} / \mathrm{s}$, with a median speed of $3.3 \mathrm{deg} / \mathrm{s}$ (Fig. 1C), which is on the low end of speed tuning for On-Off-DSGCs in mice (Weng et al., 2005) and therefore may not activate inhibitory circuits with the appropriate timing to generate null-side inhibition.

In conclusion, we demonstrated that three types of DSGCs are depolarized by retinal waves but that neither the bias in wavedirection propagation nor the direction of waves were found to correlate with DSGC spiking. These findings are consistent with the hypothesis that the directional biases induced by retinal waves are not instructive for the establishment of directional circuits. However, they do not rule out activity in all aspects of the establishment of direction selectivity. The processes of starburst amacrine cells and DSGCs cofasciculate early in the first postnatal week (Stacy and Wong, 2003; Yonehara et al., 2008), at an age during which starburst cells and DSGCs are both strongly activated by retinal waves. During the second postnatal week, preventing synaptic release from On-bipolar cells reduced the rate of synapse formation, leading to weaker connections between bipolar and ganglion cells (Kerschensteiner et al., 2009). Hence, retinal waves may provide a source of depolarization that is necessary for preserving the appropriate synapses and/or pruning the inappropriate synapses for properly wiring up directional circuits.

\section{References}

Blankenship AG, Ford KJ, Johnson J, Seal RP, Edwards RH, Copenhagen DR, Feller MB (2009) Synaptic and extrasynaptic factors governing glutamatergic retinal waves. Neuron 62:230-241.

Chan YC, Chiao CC (2008) Effect of visual experience on the maturation of $\mathrm{ON}-\mathrm{OFF}$ direction selective ganglion cells in the rabbit retina. Vision Res 48:2466-2475.

Chen M, Weng S, Deng Q, Xu Z, He S (2009) Physiological properties of direction-selective ganglion cells in early postnatal and adult mouse retina. J Physiol 587:819-828.

Efron B, Tibshirani R (1986) Bootstrap methods for standard errors, confidence intervals, and other measures of statistical accuracy. Stat Sci 1:54-75.

Elstrott J, Feller MB (2009) Vision and the establishment of direction-selectivity: a tale of two circuits. Curr Opin Neurobiol 19:293-297.

Elstrott J, Anishchenko A, Greschner M, Sher A, Litke AM, Chichilnisky EJ, Feller MB (2008) Direction selectivity in the retina is established independent of visual experience and cholinergic retinal waves. Neuron 58:499-506.

Engert F, Tao HW, Zhang LI, Poo MM (2002) Moving visual stimuli rapidly induce direction sensitivity of developing tectal neurons. Nature 419:470-475

Fried SI, Masland RH (2007) Image processing: how the retina detects the direction of image motion. Curr Biol 17:R63-R66.

Fried SI, Münch TA, Werblin FS (2002) Mechanisms and circuitry underlying directional selectivity in the retina. Nature 420:411-414.

Huberman AD, Feller MB, Chapman B (2008) Mechanisms underlying development of visual maps and receptive fields. Annu Rev Neurosci 31:479-509.

Huberman AD, Wei W, Elstrott J, Stafford BK, Feller MB, Barres BA (2009) Genetic identification of an On-Off direction-selective retinal ganglion cell subtype reveals a layer-specific subcortical map of posterior motion. Neuron 62:327-334.

Kerschensteiner D, Wong RO (2008) A precisely timed asynchronous pattern of $\mathrm{ON}$ and $\mathrm{OFF}$ retinal ganglion cell activity during propagation of retinal waves. Neuron 58:851-858.

Kerschensteiner D, Morgan JL, Parker ED, Lewis RM, Wong RO (2009)
Neurotransmission selectively regulates synapse formation in parallel circuits in vivo. Nature 460:1016-1020.

Kim IJ, Zhang Y, Yamagata M, Meister M, Sanes JR (2008) Molecular identification of a retinal cell type that responds to upward motion. Nature 452:478-482.

Li Y, Van Hooser SD, Mazurek M, White LE, Fitzpatrick D (2008) Experience with moving visual stimuli drives the early development of cortical direction selectivity. Nature 456:952-956.

McLaughlin T, Torborg CL, Feller MB, O'Leary D (2003) Retinotopic map refinement requires spontaneous retinal waves during a brief critical period of development. Neuron 40:1147-1160.

Oyster CW, Takahashi E, Collewijn H (1972) Direction-selective retinal ganglion cells and control of optokinetic nystagmus in the rabbit. Vision Res 12:183-193.

Stacy RC, Wong RO (2003) Developmental relationship between cholinergic amacrine cell processes and ganglion cell dendrites of the mouse retina. J Comp Neurol 456:154-166.

Stafford BK, Sher A, Litke AM, Feldheim DA (2009) Spatial-temporal patterns of retinal waves underlying activity-dependent refinement of retinofugal projections. Neuron 64:200-212.

Stosiek C, Garaschuk O, Holthoff K, Konnerth A (2003) In vivo two-photon calcium imaging of neuronal networks. Proc Natl Acad Sci US A 100:7319-7324.

Sun C, Warland DK, Ballesteros JM, van der List D, Chalupa LM (2008) Retinal waves in mice lacking the beta2 subunit of the nicotinic acetylcholine receptor. Proc Natl Acad Sci U S A 105:13638-13643.

Sun W, Deng Q, Levick WR, He S (2006) ON direction-selective ganglion cells in the mouse retina. J Physiol 576:197-202.

Taylor WR, Vaney DI (2002) Diverse synaptic mechanisms generate direction selectivity in the rabbit retina. J Neurosci 22:7712-7720.

Taylor WR, Vaney DI (2003) New directions in retinal research. Trends Neurosci 26:379-385.

Torborg CL, Feller MB (2005) Spontaneous patterned retinal activity and the refinement of retinal projections. Prog Neurobiol 76:213-235.

Wang CT, Blankenship AG, Anishchenko A, Elstrott J, Fikhman M, Nakanishi S, Feller MB (2007) GABA(A) receptor-mediated signaling alters the structure of spontaneous activity in the developing retina. J Neurosci 27:9130-9140.

Wang L, Rangarajan KV, Lawhn-Heath CA, Sarnaik R, Wang BS, Liu X, Cang J (2009) Direction-specific disruption of subcortical visual behavior and receptive fields in mice lacking the beta2 subunit of nicotinic acetylcholine receptor. J Neurosci 29:12909-12918.

Wei W, Elstrott J, Feller MB (2010) Two-photon targeted recording of GFPexpressing neurons for light responses and live cell imaging in the mouse retina. Nat Protoc 5:1347-1352.

Weng S, Sun W, He S (2005) Identification of ON-OFF direction-selective ganglion cells in the mouse retina. J Physiol 562:915-923.

Xu HP, Chen H, Ding Q, Xie ZH, Chen L, Diao L, Wang P, Gan L, Crair MC, Tian N (2010) The immune protein CD3zeta is required for normal development of neural circuits in the retina. Neuron 65:503-515.

Yonehara K, Shintani T, Suzuki R, Sakuta H, Takeuchi Y, NakamuraYonehara K, Noda M (2008) Expression of SPIG1 reveals development of a retinal ganglion cell subtype projecting to the medial terminal nucleus in the mouse. PLoS One 3:e1533.

Yonehara K, Ishikane H, Sakuta H, Shintani T, Nakamura-Yonehara K, Kamiji NL, Usui S, Noda M (2009) Identification of retinal ganglion cells and their projections involved in central transmission of information about upward and downward image motion. PLoS One 4:e4320.

Yoshida K, Watanabe D, Ishikane H, Tachibana M, Pastan I, Nakanishi S (2001) A key role of starburst amacrine cells in originating retinal directional selectivity and optokinetic eye movement. Neuron 30:771-780.

Zhang LL, Pathak HR, Coulter DA, Freed MA, Vardi N (2006) Shift of intracellular chloride concentration in ganglion and amacrine cells of developing mouse retina. J Neurophysiol 95:2404-2416.

Zheng JJ, Lee S, Zhou ZJ (2004) A developmental switch in the excitability and function of the starburst network in the mammalian retina. Neuron 44:851-864. 